\title{
Detección de talentos: propuesta de un instrumento para la evaluación del aspecto sicológico (CST- 40)
}

\author{
Talent detection: proposal of an instrument for the evaluation of the \\ psychological aspect (CST-40) \\ * Daniel Castro González
}

Castro, D. (2019). Detección de talentos: propuesta de un instrumento para la evaluación del aspecto sicológico (CST40). Revista Ciencias de la Actividad Física UCM, N 20(1), enero-junio, 1-13. DOI: http://doi.org/10.29035/rcaf.20.2.1

El presente estudio fue financiado mediante el Fondo Nacional para el Fomento del Deporte FONDEPORTE, del Instituto Nacional de Deportes de Chile (IND).

\begin{abstract}
RESUMEN
El propósito del presente estudio fue el diseño y validez de contenido de un instrumento de evaluación (CST-40), que permita evaluar variables sicológicas fundamentales para el rendimiento deportivo, en el ámbito de la detección de talentos deportivos (autoconfianza, concentración, control de la ansiedad, y motivación). La metodología utilizada consideró una exposición del instrumento a juicio de expertos (5), además de su aplicación experimental junto al Cuestionario de Campeones de Rushall. La muestra estuvo conformada por 115 jóvenes de 10 a 14 años de edad, damas y varones, categorizados talentos deportivos, pertenecientes a 9 disciplinas deportivas a nivel nacional. Los resultados obtenidos muestran una consistencia interna, mediante el a de Cronbash de 0,79, mientras que la correlación en función del Cuestionario de Campeones de Rushall, alcanzo un Indice de Pearson de 0,51.
\end{abstract}

\section{PALABRAS CLAVE}

Detección de talentos, factores sicológicos, rendimiento deportivo.

\begin{abstract}
This study's purpose was the design and content validity of an assessment instrument (CST-40), which allows evaluating fundamental psychological variables for sports performance, in the field of sports talent detection (self-confidence, concentration, anxiety control and motivation). The methodology used considered an exhibition of the instrument in the opinion of experts (5), in addition to its experimental application together with the Rushall Champions Questionnaire. The sample consisted of 115 young people from 10 to 14 years old, women and men, categorized sporting talents, belonging to 9 sports disciplines nationwide. The obtained results show an internal consistency, using the Cronbash a of 0.79 , while the correlation according to the Rushall Champions Questionnaire reached a Pearson Index of 0.51 .
\end{abstract}

\section{Key words}

Detection of talents, psychological factors, sports performance.

* Académico, Facultad de Educación, Universidad de Antofagasta, Chile. 


\section{PLANTEAMIENTO DEL PROBLEMA}

La identificación y selección del talento deportivo, es sin duda alguna, una estrategia a considerar para lograr un alto desempeño deportivo. En nuestro país, la ley del Deporte, 19.712, señala entre las acciones escenciales del Programa Nacional del Deporte de Alto Rendimiento: "La detección, selección y desarrollo de personas - hombres y mujeres - dotadas de talentos deportivos" (Ministerio del Interior, 2018: 4). Por su parte, las Bases de los Centros de Entrenamiento Regionales (CER), cita como objetivo general el "Desarrollar conforme a la Política Nacional de Deportes una estrategia orientada a la captación y desarrollo de los talentos con condiciones especiales para la práctica deportiva del alto rendimiento", entre sus objetivos específicos esta el de: "Confeccionar herramientas para la detección de futuros talentos deportivos" (IND, 2018).

Investigaciones realizadas en Chile concluyen la necesidad de desarrollar programas, que incluyan test de acondicionamiento nacional, utilizando procedimientos reconocidos, o bien desarrollar baterías basadas en circunstancias propias de nuestra población. Acerca del aspecto sicológico, mencionan que a pesar de la importancia que los entrenadores le asignan, al momento de la selección de talentos, este aspecto es relegado al último plano (Rojas, 2006). Castro (2008: 25-26) por su parte, concluye:

No se logran establecer los parámetros, ni se cuenta con algún instrumento que permita evaluar el aspecto psicológico, en personas con talento sin experiencia deportiva. Los instrumentos encontrados, están diseñados en función de personas con experiencia deportiva.

El estudio finaliza recomendando "Diseñar y validar un instrumento para la evaluación del aspecto sicológico, dirigido a personas sin experiencia deportiva”.

\section{Variables sicológicas fundamentales en el deporte}

Los estudios en el área de la sicología del deporte mencionan numerosas variables influyentes en el rendimiento deportivo. Sin embargo, existe una gran coincidencia sobre aquellas que son fundamentales y transversales a toda disciplina y situación deportiva, siendo las de mayor consideración la Autoconfianza, el estado de Ansiedad, la Concentración, y la Motivación.

La tendencia actual del estudio de la competitividad se enmarca en el ámbito de la motivación de logro, entendida esta como una característica de la persona humana, influyendo su conducta en variadas dimensiones de su vida laboral, social, como también deportiva, referida al deseo de ganar en situaciones interpersonales (Houston, 2002; Houston, 2005). Estudios más recientes, han descrito que los factores sociales y culturales, influyen directamente, variando en individuos de diferentes culturas (Houston, 2005). Así entendida, la competitividad se ve favorecida con la presencia de las variables mencionadas, determinando un alto grado de rendimiento.

Así, "la autoconfianza" en el deporte, representa la disposición de una persona para esforzarse en satisfacer un estándar de excelencia”, cuando se hacen comparaciones en presencia de evaluadores externos, constituyéndose en una conducta de logro en un contexto competitivo (Martens, 1990; Vealey, 1986). En cuanto al género, los hombres presentan mayor competitividad que las mujeres, estas diferencias son independientes del país o cultura al que pertenezcan (Hinsz, 2005; Remor, 2007). Por otra parte "la ansiedad", entendida como una reacción emocional ante la percepción de peligro o amenaza, se manifiesta como un conjunto de respuestas, de aspecto subjetivo y cognitivo y de carácter displacentero, como también aspectos corporales fisiológicos, caracterizados por un alto grado de activación del sistema nervioso autónomo, expresándose en conductas de tipo observables en el ámbito motor, que suelen implicar comportamientos poco ajustados y escasamente adaptativos (Salvatierra, 1998). En el contexto deportivo, hace 
referencia a una reacción de tensión relevante en situaciones de competición, en relación a la ejecución deportiva (Ortin-Montero, 2013; Hagan Jr., 2017). Desde la Teoría Multidimensional, la ansiedad competitiva en los deportistas se manifiesta en su dimensión cognitiva (reacción cognitiva ante el estado de ansiedad), ansiedad somática (nivel de activación autónoma) influyendo sobre la autoconfianza (Martens, 1990; Pinto, 2013).

Existe una reciproca relación entre autoconfianza y ansiedad. La interpretación facilitadora de los síntomas de ansiedad, son consecuencia de unos niveles bajos de los mismos y posiblemente combinado con altos niveles de autoconfianza (Lundqvist, 2011; Mullen, 2009). La autoconfianza parece actuar como reguladora de los síntomas de ansiedad en deportistas de alto rendimiento, donde altos niveles de autoconfianza y bajas manifestaciones de ansiedad proporcionan una percepción facilitadora de la competencia (Mellalieu, 2006).

"La concentración" involucra diferentes elementos, que en definitiva se traducen en un estado de atención, determinado por la duración de la tarea en situación de competición o entrenamiento (García, 2001; González, 2007), permiendo la focalización de la tarea a realizar. Los aspectos más influyentes para lograr una eficiente concentración son:

1. El conocer las señales específicas que deben ser atendidas.

2. Mantener el foco de la atención en las señales relevantes a la tarea.

3. La habilidad para cambiar el foco de la atención, de acuerdo a las circunstancias.

4. La habilidad para "re-concentrarse", cuando ocurra algún tipo de distracción.

5. El control de los pensamientos irrelevantes y ajenos a la tarea.

6. La Inhibición de estímulos distractores, presentes durante la situación deportiva.
En cuanto a "la motivación", su comprensión en el deporte ha evolucionado desde modelos mecanicistas, hacia aquellos de carácter social y cognitivo. El marco teórico que sustenta la motivación en la actividad físico-deportiva, está basado en la teoría de la autodeterminación (TAD), la cual analiza el grado en que las conductas humanas son de origen volitivo o auto-determinadas (García-Calvo, 2011; Luckwü, 2011; Moreno, Cervelló, y González, 2007). Así entendida, la autodeterminación es considerada una manifestación intrínseca de la motivación humana, el grado en que las personas realizan sus acciones en forma voluntaria y por propia elección, con un alto nivel de reflexión y compromiso (Carratalá, 2004; Deci, 2002, 2013). En este contexto destaca el rol del entrenador sobre el nivel motivacional de los deportistas, debiendo transmitir claramente el significado de las actividades que realizan, apoyando la libertad y la autonomía, que trasciendan hacia los procesos de toma de decisiones (Balaguer, 2015; Fabra, 2018; Fiorese, 2017).

\section{Instrumentos de evaluación relacionados}

Diversos instrumentos utilizados para evaluar el aspecto sicológico del rendimiento deportivo, coinciden en citar los factores antes descritos. Así, el Cuestionario de Características sicológicas relacionadas con el rendimiento deportivo (CPRD), (Gimeno, 2001), de 55 ítems con respuestas tipo Likert de 5 niveles, considera los siguientes factores:

- El control del stress (ansiedad).

- La evaluación del rendimiento deportivo.

- La motivación.

- La habilidad mental.

- La cohesión de equipo.

Por otra parte, el Cuestionario COMPETITIVIDAD-10 (Remor, 2007), consta de 10 ítems, también con respuestas tipo Likert de 3 niveles, aplica un análisis sobre las dimensio- 
nes de motivación de éxito $(\mathrm{Me})$ y la motivación para evitar el fracaso (Mef). Considera las siguientes variables:

- Aspectos socio-demográficas: género, edad, tipo de práctica deportiva.

- Motivación de éxito.

- Motivación para evitar el fracaso.

- Grado de competitividad.

- Autovaloración subjetiva de la competitividad.

El Cuestionario de Ansiedad Precompetitivo (CSAI-2R), (Andrade, 2007; Martens, 1990), lo componen 17 ítems, con 4 niveles de respuestas tipo Likert, considera las subescalas de:

- Ansiedad cognitiva.

- Ansiedad somática.

- Autoconfianza.

El Cuestionario de Rasgos Sicológicos en Deportistas (PAR-P1), (Serrato, 2006): considera 45 Items, presentando un índice de consistencia interna (Alfa de Cronbach) con valores superiores a 0.70 en todas sus escalas y el conjunto de la prueba. Los factores que evalúa son:

- Confianza.

- Motivación.

- Concentración.

- Sensibilidad emocional.

- Imaginación.

- Actitud positiva.

- Reto.

El Inventario Psicológico de Ejecución Deportiva (IPED) (Raimundi, 2016): Mediante 42 ítems y 5 opciones de respuesta tipo Liker, el IPED en su versión española, ha sido aplicado a un gran número de practicantes de distintas modalidades deportivas, Su índice de fiabilidad va desde 0,70 tanto en sus factores como en su integralidad. (Hernández-Mendo, A., Morales V., y Peñalver I., 2014; HernándezMendo, 2006), es una herramienta muy confiable y precisa a la hora de evaluar las habilidades psicológicas "en deportistas". Considera los siguientes factores:
- La autoconfianza.

- El control de Afrontamiento Negativo.

- El control Atencional.

- El control Viso-Imaginativo.

- El nivel Motivacional.

- El control de Afrontamiento Positivo.

- El control Actitudinal.

El Cuestionario de Características de los Campeones (CCC), (Rushall, 1988; 1994; 2001), consta de 100 ítems con respuestas dicotómicas (presencia-ausencia), considera indicadores consistentes y comunes presentes en deportistas de nivel mundial. Un puntaje de 85 pts. es bueno en adultos y 75 pts. en deportistas jóvenes. En estudio con jóvenes talentos "con experiencia deportiva sistemática" en Chile, el CCC arrojo una mediana de 69,8 puntos (Castro, 2008). Las dimensiones que estima son:

- La relación con otros deportistas (10).

- La relación con su entrenador (7).

- La reacción ante el fracaso (5).

- Actitud ante el entrenamiento (7).

- La valoración de su actividad deportiva (13).

- La focalización en su actividad deportiva (10).

- Factores precompetitivos (17).

- Factores propios de la competencia deportiva (11). (20).

- Motivación por la práctica deportiva

* En paréntesis la cantidad de ítems para cada dimensión.

\section{OBJETIVOS DE INVESTIGACIÓN}

\section{Objetivo General}

- Diseñar y validar un instrumento de evaluación del aspecto sicológico dirigido a personas damas y varones, de 10 a 14 años de edad, sin experiencia deportiva, en el contexto de una detección de talentos deportivos en Chile. 


\section{Objetivos Específicos}

- Diseñar la estructura de contenido del instrumento CST-40, definiendo factores, dimensiones, Items e indicadores de respuesta.

- Validar el contenido del instrumento CST40, mediante 2 acciones:

a. Exposición del cuestionario CST-40 a juicio de 5 expertos.

b. Aplicación experimental de los cuestionarios CST-40 y CCC a una muestra de jovenes talentos deportivos de 10 a 14 años hombres y mujeres.

\section{Diseño metodológico}

\section{Hipótesis}

La aplicación del instrumento CST-40, permite evaluar características sicológicas fundamentales para el rendimiento deportivo (Autoconfianza, Concentración, Control de la Ansiedad y Motivación), en jóvenes hombres y mujeres de 10 a 14 años, sin experiencia deportiva.

\section{Tipo de Investigación}

Trabajo de desarrollo, cuasi-experimental, de temporalidad transeccional.

\section{Muestra}

De tipo aleatoria intencionada, conformada por 115 deportistas, damas y varones de 10 a 14 años de edad. El criterio de inclusión considero la categorización de talento deportivo perteneciente a escuelas federadas con destacados logros a nivel internacional (Tabla 1).
Tabla 1

Muestra: Distribución por género y deportes.

\begin{tabular}{lccc}
\hline Deporte & Damas & Varones & Total \\
\hline Atletismo & 4 & 3 & 7 \\
\hline Balonmano & 8 & 11 & 19 \\
\hline Basquetbol & 10 & 13 & 23 \\
\hline Fútbol & --- & 20 & 20 \\
\hline Halterofilia & 1 & 6 & 7 \\
\hline Hockey-patín & 6 & 4 & 10 \\
\hline Karate & 4 & 3 & 7 \\
\hline Taekwondo & --- & 9 & 9 \\
\hline Tenis & 2 & 11 & 13 \\
\hline Total & 35 & 80 & 115 \\
\hline
\end{tabular}

\section{PROCEDIMIENTO}

Para el diseño del CST-40, se realizó en primer lugar una exhaustiva indagación y análisis de estudios e instrumentos de evaluación existentes a nivel internacional, relacionados con los aspectos sicológicos del rendimiento deportivo (Andrade, 2017; Castro, 2008; Gimeno, 2001; Guillén, 2007; Ortin, 2013; Pozo, 2007; Raimundi, 2016; Ramis, 2013; Remor, 2007; Rushall, 2001; Serrato, 2006). Los factores fueron definidos de acuerdo a la mayor frecuencia presente en los instrumentos consultados, considerando aquellas variables psicológicas básicas del deporte (Autoconfianza, Concentración, Control de la Ansiedad y Motivación). La definición de factores fue definida analógicamente según las siguientes dimensiones:

- Situaciones del quehacer escolar.

- Actitud hacia la clase de educación física y deportes.

- Influencia de la familia y de los pares sobre las actividades de los evaluados.

Los ítems se elaboraron en base a los enunciados en el CCC, simplificando la redacción, disminuyendo la cantidad, considerando el propósito de evaluar a personas sin experiencia deportiva. Se definieron 10 Items por factor, en forma aleatoria de acuerdo al siguiente prorrateo: 
Tabla 2

Distribución de items: prorrateo del factor autoconfianza.

\begin{tabular}{l}
\hline Items \\
\hline 4. Me gusta ser el mejor en todo lo que hago. \\
\hline 8. Me enojo cuando bajo mi promedio de notas. \\
\hline 12. Me esfuerzo para ser de los mejores en mi curso. \\
\hline 16. Busco superarme en todo lo que hago. \\
\hline 20. En todo lo que hago, quiero ser mejor que el año pasado. \\
\hline 24. Superare a mis compañeros(as) que tienen mejores notas que yo. \\
\hline 28. Cuando algo no me sale, practico más para poder mejorar. \\
\hline 32. Me gusta competir con mis compañeros en la clase de educación física. \\
\hline 36. Al competir demuestro que me la puedo. \\
\hline 40. solo estaré conforme cuando este entre los mejores. \\
\hline
\end{tabular}

\section{Tabla 3}

Distribución de items: prorrateo del factor concentración.

\begin{tabular}{l}
\hline Items \\
\hline 2. En la clase de educación física, observo a los demás y luego realizo los ejercicios. \\
\hline 6. En las pruebas del colegio estoy siempre concentrado. \\
\hline 10. Cuando juego estoy pendiente de lo que hacen mis rivales. \\
\hline 14. Cuando juego, Identifico las debilidades de mi rival. \\
\hline 18. En disertaciones del colegio, me fijo en lo bueno y lo malo de mis compañeros. \\
\hline 22. En las pruebas, analizo detenidamente las preguntas y luego decido la respuesta. \\
\hline 26. En televisión veo deporte para aprender más. \\
\hline 30. Leo sobre deporte para aprender más. \\
\hline 34. Los partidos de Futbol se pierden por errores propios. \\
\hline 38. En la clase de Educación Física, imito a los que realizan bien los ejercicios. \\
\hline
\end{tabular}

Tabla 4

Distribución de items: prorrateo del factor control de la ansiedad.

\begin{tabular}{l}
\hline Items \\
\hline 3. Siempre mantengo la calma en toda actividad que realizo. \\
\hline 7. La noche anterior a una prueba duermo tranquilo. \\
\hline 11. Cuando realizo pruebas en el colegio, controlo mis nervios. \\
\hline 15. En las pruebas de educación física me va bien. \\
\hline 19. No me pongo nervioso cuando mi familia está presente en mis actividades. \\
\hline 23. durante los controles de educación física mantengo la calma. \\
\hline 27. Al apurarse las cosas salen mal. \\
\hline 31. Con paciencia las cosas salen mejor. \\
\hline 35. Espero con ganas que llegue el día de la clase de Educación Física. \\
\hline 39. Durante la realización de mis actividades, sé que lo hare bien.
\end{tabular}


Tabla 5

Distribución de items: prorrateo del factor motivación.

\begin{tabular}{l}
\hline Items \\
\hline 1. Me siento muy bien después de hacer deporte. \\
\hline 5. Al hacer deporte me divierto. \\
\hline 9. Me gusta invitar a mis amigos a hacer deporte. \\
\hline 13. en las clases de educación física me entretengo muchísimo. \\
\hline 17. Me gusta que mi familia me acompañe y apoye en las actividades deportivas. \\
\hline 21. Los fines de semana hago deporte. \\
\hline 25. Me entretengo más haciendo deporte que otras actividades. \\
\hline 29. En mi tiempo libre prefiero hacer deporte que quedarme en casa. \\
\hline 33. Me gusta que mi familia y amigos destaquen mis logros. \\
\hline 37. Yo quiero hacer deporte porque así soy feliz. \\
\hline
\end{tabular}

El formato de respuestas se estructuro en escala de Likert, con 3 opciones a las que se asignó un puntaje que facilitara el tratamiento estadístico, de acuerdo a la siguiente equivalencia:

- Nunca (0 punto); - A veces (0,5 punto); Siempre (1 punto).

\section{Juicio de expertos}

El CST-40 fue expuesto a 5 profesionales con experticia en Metodología de la Investigación, Psicología General, Psicología Deportiva, y Sicopedagogía, considerando las siguientes dimensiones de análisis:

- La estructura de los Items que componen el instrumento.

- El tipo de respuesta propuesto.

- Los factores (variables sicológicas) consideradas en el CST-40.

- La propuesta de considerar las dimensiones escolar y familiar.

- Una opinión personal abierta acerca del CST-40.

\section{Aplicación experimental}

El cuestionario CST-40, se aplicó junto al CCC, a la muestra de jovenes talentos deporti- vos, dicha acción permitió conocer la dinamica de los factores, la consistencia interna, y la correlación entre ambos instrumentos.

\section{Tratamiento estadístico}

Los datos fueron analizados, mediante el software SPSS 22.0, versión en español, realizando un análisis descriptivo multivariado acerca de los estadísticos de mediana, desviacion estandar, Indice de consistencia Alfa de Cronbach y de correlación de Pearson entre ambos cuestionarios.

\section{RESULTADOS}

La revisión bibliográfica, permitio identificar aquellos factores de mayor presencia en los instrumentos de evaluación consultados, siendo dichos factores expuestos a la segunda etapa de juicio de expertos, junto a los demás aspectos estructurales y dimensiones, obteniendo una validez de contenido Alfa de Cronbach de 0,63.

Mediante la aplicación experimental de CST-40 y CCC, se obtuvo los valores por deporte (Tabla $\left.n^{\circ} 6\right)$. Con el fin de facilitar la comparación, se incluyó los valores factorizados del CST-40, en función de la relación de equivalencia cuantitativa directa $(1: 2,5)$. 
Tabla 6

Respuestas por deportes: cuestionario CST-40 y

CCC (mediana y DS).

\begin{tabular}{lccccc}
\hline & \multicolumn{2}{c}{ CST-40 } & CST-40(FACT.) & \multicolumn{2}{c}{ Rushall } \\
\hline Deporte & Mediana & DS & Mediana & Mediana & DS \\
\hline Tenis & 29,5 & 3,7 & 73,8 & 77,0 & 9,3 \\
\hline Basquetbol & 28,3 & 3,7 & 70,8 & 82,0 & 9,3 \\
\hline Hockey P. & 28,8 & 6,5 & 72,0 & 77,5 & 16,8 \\
\hline Karate & 29,0 & 2,3 & 72,5 & 81,0 & 9,1 \\
\hline Balonmano & 28,0 & 3,2 & 70,0 & 81,0 & 8,9 \\
\hline Halterofilia & 28,5 & 3,7 & 71,3 & 81,0 & 12,0 \\
\hline Atletismo & 30,0 & 0,9 & 75,0 & 81,0 & 5,6 \\
\hline Fútbol & 32,0 & 2,8 & 80,0 & 83,0 & 7,6 \\
\hline Taekwondo & 30,0 & 3,1 & 75,0 & 81,0 & 6,4 \\
\hline General & 29,5 & 3,9 & 73,8 & 81,0 & 10,5 \\
\hline
\end{tabular}

Las respuestas por factor del CST-40, los valores en autoconfianza, control de la ansiedad y motivación (8 a 8,5-1,2DS), a excepción del factor concentración (6 - 1,4DS), situación similar presente en los valores obtenidos por deporte, a excepción del Tenis (8-1,4DS) (Tabla 7).

Tabla 7

Respuestas por deportes: cuestionario CST-40 y CCC (mediana y DS).

\begin{tabular}{lcccccccc}
\hline & \multicolumn{2}{c}{ Autoconfianza } & Concentración & \multicolumn{2}{c}{ C. Ansiedad } & \multicolumn{2}{c}{ Motivación } \\
\hline Deporte & Med. & DS & Med. & DS & Med. & DS & Med. & DS \\
\hline Tenis & 8 & 1,4 & 8 & 1,4 & 8 & 0,8 & 7,5 & 1,5 \\
\hline Basquetbol & 8,5 & 1,2 & 5,5 & 1,2 & 8 & 1,1 & 8 & 1,6 \\
\hline Hockey Pat. & 8,3 & 1,5 & 6,3 & 1,8 & 9 & 2,6 & 8 & 1,9 \\
\hline Karate & 7,5 & 1,3 & 7 & 1,3 & 7,5 & 1 & 7,5 & 0,9 \\
\hline Balonmano & 8 & 1,1 & 5 & 1,4 & 7,5 & 0,7 & 8 & 1,2 \\
\hline Halterofilia & 7 & 1,6 & 5 & 1,7 & 8 & 0,5 & 9 & 1,3 \\
\hline Atletismo & 8,5 & 0,9 & 6 & 0,6 & 8,5 & 0,6 & 8 & 0,6 \\
\hline Fútbol & 9 & 0,9 & 6,8 & 1,1 & 8 & 0,8 & 8,3 & 1 \\
\hline Taekwondo & 8 & 1,3 & 6 & 1,5 & 8 & 0,8 & 8 & 1 \\
\hline General & 8,5 & 1,2 & 6,0 & 1,3 & 8 & 1,1 & 8 & 1,3 \\
\hline
\end{tabular}

La consistencia Interna de CST-40, alcanza un $a$ de Cronbach de 0,80, mientras que por factor, los valores obtenidos van de 0,43 a 0,64 , La correlación obtenida entre CST-40 respecto de CCC fue positiva, 0,51 (Tabla 8). 
Tabla 8

Consistencia interna:(a) de Cronbach e índice de Pearson.

\begin{tabular}{llcc}
\hline \multirow{2}{*}{ Cuestionario } & \multicolumn{1}{c}{$\begin{array}{c}\text { a de } \\
\text { Cronbach }\end{array}$} & $\begin{array}{c}\text { Coeficiente de } \\
\text { Pearson }\end{array}$ \\
\hline \multirow{4}{*}{ Factores } & CST-40 & 0,80 & 0,51 \\
\cline { 2 - 3 } & Rushall & 0,86 & \\
\cline { 2 - 3 } & Autoconfianza & 0,60 \\
\cline { 2 - 3 } & Concentración & 0,56 \\
\hline & $\begin{array}{l}\text { Control de la } \\
\text { Ansiedad }\end{array}$ & 0,43 \\
\cline { 2 - 3 } & Motivación & 0,64 \\
\hline
\end{tabular}

La dispersión de valores entre ambos cuestionarios, presenta una tendencia lineal coincidente al índice positivo de correlación encontrado entre ambos instrumentos de evaluación (Gráfico 1).

\section{Gráfico 1}

Dispersión de valores de CST-40/CCC (valores máximos 40/100 puntos).

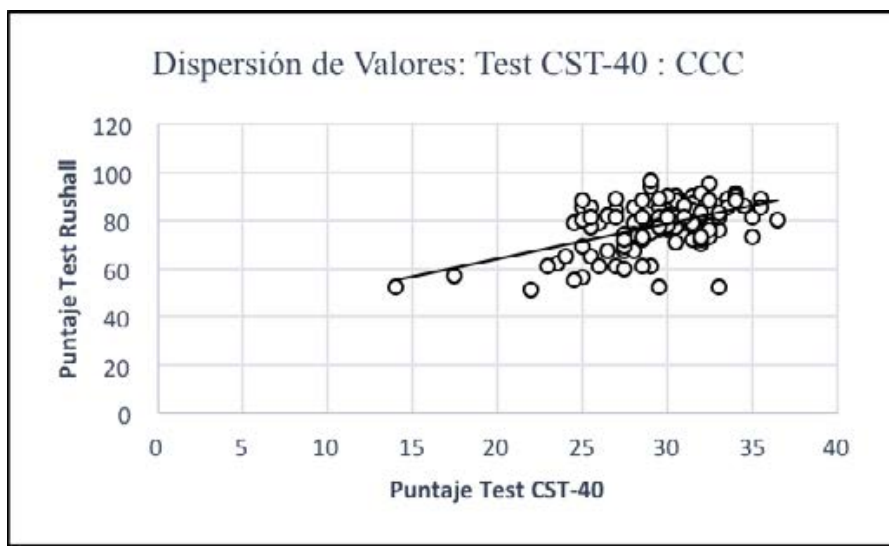

\section{DISCUSIÓN}

Siendo el propósito de la investigación poder contar con un instrumento que permita evaluar el aspecto sicológico en personas jóvenes sin experiencia práctica del deporte, en el contexto de una detección de talentos deportivos, la indagación acerca de los instrumentos de evaluación existentes, permitieron señalar a los factores de Autoconfianza, Concentración, Control de la ansiedad y Motivación como los más emergentes y fundamentales para la práctica competitiva del deporte (Andrade, 2017; Castro, 2008; Gimeno, 2001; Moreno, et al., 2007; Raimundi, 2016; Remor, 2007; Rushall, 2001; Serrato, 2006). Dichos factores deben ser considerados e integrados en el diseño y definición de estrategias de enseñanza, tanto en el ámbito de la formación deportiva, como también en la formación docente de la actividad física, incluyéndolas como contenidos integrantes de los aprendizajes planteados en las asignaturas referidas a deportes.

La validación mediante juicio de experto confirma los factores enunciados, en su concepto de fundamentales y transversales a la práctica deportiva competitiva, en diferentes especialidades. También da validez a la propuesta análoga de las dimensiones, en consideración de la población objeto de estudio (sin experiencia deportiva) $(0,63)$.

La aplicación experimental permitió obtener la consistencia interna del CST-40 $(0,80$ frente a 0,86 de CCC), como tambien de los 4 
factores evaluados (Autoconfianza 0,60; Concentración 0,43; Control de la Ansiedad 0,56; Motivación 0,64).

Si bien los valores absolutos obtenidos en CCC son más altos (81,0 versus CST-Factorizado 73,8 ), Esto probablemente se explique por la característica de la muestra (talentos deportivos), siendo los ítems de CCC mas concurrentes a dicha condición, siendo válida dicha observación al comparar los resultados obtenidos del CCC, con los de Castro (2008), en donde la muestra fue más heterogénea $(81,0$ - 69,8). Al respecto el índice de correlación positiva alcanzado (Coeficiente de Pearson 0,51 ), junto a la gráfica lineal de dispersión son concluyentes.

\section{CONCLUSIONES}

Los resultados obtenidos en base a la indagación bibliográfica y la aplicación de juicio de expertos, logra dar validez de contenido al Cuestionario CST-40.

En segundo término, la aplicación experimental realizada, permite establecer la consistencia interna del Cuestionario CST-40 como también de sus factores.

El estudio permite contar con un instrumento para evaluar la presencia de variables sicológicas fundamentales en procesos de detección de talentos deportivos en jóvenes mujeres y hombres de 10 a 14 años en nuestro país.

Se recomienda realizar investigaciones que permitan dar validez de constructo a CST-40, como la definición de percentiles y escalas de apreciación, en función de los diferentes factores, rangos de edad, y género.

\section{REFERENCIAS BIBLIOGRÁFICAS}

Andrade Fernández, E. M., Lois Río, G., \& Arce Fernández, C. (2007). Propiedades psicométricas de la versión española del Inventario de Ansiedad Competitiva CSAI-2R en deportistas. Psicothema, 19(1), 150-155. www.psicothema.com

Balaguer, I., Castillo, I., Ródenas, L., Fabra, P., y Duda, J. L. (2015). Los entrenadores como promotores de la cohesión del equipo. En Cuadernos de Psicología del Deporte, 15(1), 233-242.

Castro, D., Torres, E. (2008). Aplicación Metodológica Para Detectar Talentos en Atletismo: Regiones Metropolitana y Antofagasta, Chile. Revista de las ciencias de la actividad física del Instituto Nacional de Deportes, 3,14-30, ISSN: 0718-4492

Carratalá, E. (2004). Análisis de la teoría de las metas de logro y de la autodeterminación en los planes de especialización deportiva de la Generalitat Valenciana. Tesis Doctoral, Universidad de Valencia. España.

Deci, E. L., Ryan, R.M. (2002). Handbook of self-determination research. University of Rochester Press. Rochester, New York.

Deci, E. L., Ryan, R.M., Guay, F. (2013). Selfdetermination theory and actualization of human potential. En: Theory driving research: new wave perspectives on self process human development, 109-133.

Fabra, P. y cols. (2018). Versión Española del Sistema de Observación del Clima Motivacional Multidimensional (MMCOS): Fiabilidad y Evidencias de Validez. En Revista de Psicología del Deporte, 27, 11-22. https://www.rpdonline.com/article/view/v27-n1-fabra$\underline{\text { balaguer-tomas-etal }}$ 
Fiorese, L. y cols. (2017). Asociación entre la motivación y la cohesión del grupo en el fútbol profesional: ¿la relación entrenador-atleta es un factor determinante? Revista de Psicología del Deporte, 27(4), 51-57. https://www.rpd-online.com/ article/view/v27-s1-fiorese-contessotopizzo-etal

García-Calvo, T., Sánchez P., Leo F., Sánchez D., Amado D. (2011). Incidencia de la Teoría de Autodeterminación sobre la persistencia deportiva.(Incidence of Self-Determination Theory of sport persistence). RICYDE. Revista Internacional de Ciencias del Deporte, 7(25), 266-276. DOI: 10.5232/ricyde

García, F. (2001). Tenis y Concentración de la Atención. Revista Digital EF Deportes, 7(40). Recuperado de: http://www.efdeportes.com

Gimeno, F., Buceta, J.M. y Pérez Llantada, M. (2001). El cuestionario características psicológicas relacionadas con el rendimiento deportivo (CPRD): Características psicométricas. Análise Psicológica, 19(1), 93-113.

González, J. (2007). Herramientas Aplicadas al desarrollo de la Concentración en el Alto Rendimiento Deportivo. Cuadernos de psicología del deporte, 7(1), 61-70. http://www.redalyc.org/ pdf/2270/227017553004.pdf

Guillén, G. Félix, (2007). Motivos de participación deportiva en natación competitiva, en niños y jóvenes. Cuadernos de Psicología del Deporte, 7(2), 59-74. Universidad de Murcia. España. http://www.redalyc.org/pdf/2270/227017599004.pdf

Hagan Jr., J. E., Pollmann, D. y Schack, T. (2017). Interaction between gender and skill on competitive state anxiety using the time-to event paradigm: ¿What roles do intensity, direction, and frequency dimensions play?. Frontiers in Psychology, 8, 692. doi:10.3389/ fpsyg.2017.00692
Hernández-Mendo, A. (2006). Cuestionario para la evaluación psicológica de la ejecución deportiva: estudio complementario entre TCT y TRI. Revista de Psicología del Deporte, 15, 71-93

Hernández-Mendo, A., Morales V., y Peñalver I. (2014). Replicación de las propiedades psicométricas del Inventario Psicológico de Ejecución Deportiva. Revista de Psicología del Deporte. 23(2), 311-324. http://www.redalyc.org/ pdf/2351/235131674011.pdf

Hinsz, V.B. y Jundt, D.K. (2005) Exploring Individual Differences in a GoalSetting Situation Using the Motivational Trait Questionnaire. Journal of Applied Social Psychology, 35(3), 551-571.

Houston, J., Harris, P., McIntire, S. y Francis, D. (2002a). Revising the competitiveness index using factor analysis. Psychological Reports, 90(1), 31-34.

Houston, J. M., Harris, P. B., Moore, R., Brummett, R. y Kmetani, H. (2005). Competitiveness among Japanese, Chinese, and American undergraduate students. Psychological Reports, 97(1), 205-212.

IND (2018). Programa de Entrenamiento Regional. Instituto Nacional de Deportes. Recuperado de: http://www.ind.cl/rendimiento-deportivo/cer/

Lundqvist, C.; Kenttä, G., \& Raglin, J.S. (2011). Directional anxiety responses in elite and sub- elite young athletes: intensity of anxiety symptoms matters. Scandinavian journal of medicine \& science in sports, 21(6), 853-862.

Luckwü R., Guzmán J. (2011). Deportividad en balonmano: un análisis desde la Teoría de la Autodeterminación. Revista de Psicología del deporte, 20(2), 0305-320. https://www.rpd-online.com/article/ view/948 
Martens, R., Burton, D., Vealey, R. S., Bump, L. A. y Smith, D. E. (1990). Competitive Anxiety in Sport. Champaign IL: Human Kinetics Publishers.

Mellalieu, S. D.; Neil, R., \& Hanton, S. (2006). Self-Confidence as a Mediator of the Relationship Between Competitive Anxiety Intensity and Interpretation. Research Quarterly for Exercise \& Sport, 77(2), 263-270.

Ministerio del Interior (2018). Ley del Deporte. Recuperado de http://bcn.cl/1uvw0

Moreno, J., Cervelló, E. \& González, D. (2007). Analizando la motivación en el deporte: un estudio a través de la teoría de la autodeterminación. Apuntes de Psicología, 25(1), 35-51.

Mullen, R.; Lane, A., \& Hanton, S. (2009). Anxiety symptom interpretation in highanxious, defensive high-anxious, low-anxious and repressor sport performers. Anxiety, Stress \& Coping, 22(1), 91-100.

Ortín-Montero, F. J., De la Vega, R. y Gosálvez-Botella, J. (2013). Optimismo, ansiedad-estado y autoconfianza en jóvenes jugadores de balonmano. Anales de Psicología, 29(3), 637-641. doi:10.6018/ analesps.29.3.175751

Pinto, M. F. y Vázquez, N. (2013). Ansiedad estado competitiva y estrategias de afrontamiento: su relación con el rendimiento en una muestra argentina de jugadores amateurs de golf. Revista de Psicología del Deporte, 22, 47-52.

Pozo, A. (2007). Intensidad y dirección de la ansiedad competitiva y expectativas de resultados en atletas y nadadores. Revista de psicología del deporte, 16(2), 0137-150. https://core.ac.uk/download/pdf/13295960.pdf
Raimundi M.J., Reigal, R., y Hernández Mendo, A. (2016). Adaptación argentina del Inventario Psicológico de Ejecución Deportiva (IPED): validez, fiabilidad y precisión. Cuadernos de Psicología del Deporte, 16(1), 211-222.

Ramis, 0., Torregrosa M., Cruz, J. (2013). Revisitando a Simón \& Martens: la ansiedad competitiva en deportes de iniciación. Revista de Psicología del Deporte. 22(1), 77-83. https://www.rpd-online.com/ article/view/v22-n1-ramis-torregrosacruz

Remor, E. (2007). Propuesta de un cuestionario breve para la evaluación de la competitividad en el ámbito deportivo: Competitividad-10. Revista de psicología del deporte, 16(2). 167-183.

Rojas, M., Serrano, C., Tobar, R. y Vargas. C. (2006). Criterios básicos para la detección, selección y seguimiento de talentos deportivos en Chile. (tesis de pregrado). Universidad Metropolitana de Ciencias de la Educación, Santiago, Chile.

Rushall, B.S., (1988). Características conductuales de los campeones. Apunts Medicina de l"Esport (Castellano), 25(096), 7582. Recuperado de http://www.apunts. org/es-pdf- X0213371788050131

Rushall, B. S. (1994). Champion characteristics. Carlile Coaches' Forum, 1(5), 1-2. Recuperado de https://coachsci.sdsu. edu/csa/vol33/rushall5.htm

Rushall, B. (2001). Woorkbook for think and act like a champion. https://coachsci. sdsu.edu/thinkact/control/workbook. pdf

Salvatierra, N. y Tovar M., (1998). Predicción de la Ansiedad en sujetos varones en el ámbito deportivo y su relación con la modalidad deportiva, el nivel académico y tipo de deporte.Revista española de medicina de la educación física y el deporte 7, 205-216. 
Serrato, L. (2006). Revisión y estandarización de la prueba elaborada para evaluar rasgos psicológicos en deportistas (PAR-P1) en un grupo de deportistas de rendimiento en Colombia. Cuadernos de Psicología del Deporte, 6(2), 6784. Ihttps://revistas.um.es/cpd/article/ view/113891

Vealey, R. S. (1986). Conceptualization of sport-confidence and competitive orientation: Preliminary investigation and instrument development. Journal of sport psychology, 8(3), 221-246.

\section{Dirección para correspondencia}

Daniel Castro González

Magíster en Alto Rendimiento Deportivo.

Universidad de Antofagasta, Chile.

Angamos 601, Antofagasta, Chile.

Contacto:

daniel.castro@uantof.cl

Recibido: 19-11-2018

Aceptado: 20-06-2019 